\title{
Modulation of the ERK1/2-MMP-2 pathway in the sclera of guinea pigs following induction of myopia by flickering light
}

\author{
MAN SHE ${ }^{1},{\text { BING } \mathrm{LI}^{2}, \text { TAO LI }}^{1}$, QIANQIAN HU ${ }^{1}$ and XIAODONG ZHOU ${ }^{1}$ \\ ${ }^{1}$ Department of Ophthalmology; ${ }^{2}$ Central Laboratory, Jinshan Hospital of Fudan University, Shanghai 201508, P.R. China
}

Received November 16, 2019; Accepted January 8, 2021

DOI: $10.3892 /$ etm.2021.9802

\begin{abstract}
It has been shown that flickering light can affect the development of eyeballs. However, the exact mechanism remains unclear. The ERK1/2-MMP-2 pathway is a classic pathway involved in the modulation of the extracellular matrix (ECM) in cancer tissues. However, to the best of our knowledge, the role of this pathway in modulating the scleral ECM in myopia has not been previously examined. The present study aimed to determine the effects of the ERK1/2-MMP-2 pathway on the formation of flickering light-induced myopia (FLM). Guinea pigs were raised under illumination at a flash rate of $0.5 \mathrm{~Hz}$ for 6 weeks to induce FLM. Peribulbar injections of dimethylsulfoxide or PD98059 (an inhibitor of phospho-ERK1/2) were administered starting at the third week of FLM modeling. Refraction was measured prior to and following treatments. The thickness of the posterior sclera (PS) was measured under a light microscope following H\&E staining. The mRNA levels of MMP-2 were detected by the reverse transcription-quantitative PCR assay. The expression levels of MMP-2 and ERK1/2 were assayed by western blot and immunohistochemical analyses. Following 6 weeks of treatment, the refraction of the FLM group became more myopic compared with that of the control group, while PD98059 treatment inhibited the changes noted in the refraction. A marked reduction in the thickness of PS was observed in the FLM group, while PD98059 inhibited the remodeling of PS. In addition, the expression levels of MMP-2 and protein levels of phospho-ERK1/2 were increased in the FLM group, while PD98059 significantly inhibited MMP-2 mRNA and protein levels. These results indicated that ERK1/2-MMP-2 may be involved in the formation of FLM in guinea pigs by regulating the remodeling of $\mathrm{PS}$.
\end{abstract}

Correspondence to: Professor Xiaodong Zhou, Department of Ophthalmology, Jinshan Hospital of Fudan University, 1508 Longhang Road, Shanghai 201508, P.R. China

E-mail: xdzhou2013@126.com

Key words: myopia, flickering light, ERK1/2, MMP-2, PD98059

\section{Introduction}

In recent years, the increasing usage of electronic equipment, such as computers, television and electronic tablets, has become widespread in adolescents and even in pre-school children (1). The flickering light emitted from the screen of electronic equipment can cause numerous side effects. Therefore, studies on the impact of the flickering light on vision have attracted increasing attention. In 1989, Cremieux et al (2) demonstrated a myopic shift in cats reared with exposure to strobe light at $2 \mathrm{~Hz}$. Similar results were noted in C57BL/6 mice by Yu et al (3). In addition, our previous study demonstrated that stroboscopic illumination at a flash rate of $0.5 \mathrm{~Hz}$ led to myopia and increased axial length in guinea pigs (4-6). Although flickering light is a repeatable stimulus that affects the refraction of the eyeballs, the mechanism of its action remains unclear. Therefore, studies on signaling pathways participating in the development of flickering light-induced myopia (FLM) are necessary.

The axial growth that occurs during myopia is associated with extracellular matrix (ECM) remodeling in posterior sclera (PS), which is regulated by genetic and environmental factors (7). Among these factors, the balance between MMP-2 and tissue inhibitor of metalloproteinases 2 (TIMP2) enzymes plays a key role in the ECM remodeling process of the sclera $(8,9)$. It has been shown that MMP-2 expression is upregulated during myopia and downregulated during the reversal of this process in tree shrews (10), guinea pigs (11) and chicks (12). Furthermore, the expression levels of MMP-2 in vitreous and aqueous humor were increased in patients with high myopia (13), such that the ratio of MMP-2/TIMP2 in aqueous humor was higher in those patients compared with that in normal subjects (14). Therefore, the levels of MMP-2 play an important role in the development of eyeballs. However, whether scleral remodeling is involved in the formation of FLM by the ERK1/2-MMP-2 pathway, to the best of our knowledge, remains unknown.

Among the regulators of MMP-2, ERK is an important molecule. ERK is a subfamily of MAPKs. These enzymes are consecutively activated as follows: MAPK kinase kinase activates MAPK kinase, which in turn activates MAPK. The ERK family includes the enzymes ERK1-ERK8. Among these proteins, ERK1 and ERK2 are the most thoroughly studied (15). ERK proteins are widely expressed and are involved in a number of physiological 
processes including meiosis and late mitotic function in different cells. Xie et al (16) demonstrated that ERK1/2 mRNA expression was gradually increased in the visual cortex of rats during the critical period from postnatal day 21 (P21) to P45 with a peak at P45. Its level subsequently declined to a level parallel to that noted on P21, indicating that ERK1/2 may be involved in the regulation of developmental plasticity of the visual cortex during the critical period. Barathi et al (17) demonstrated that the proliferative responses of scleral fibroblasts (SFs) to muscarinic receptor stimulation were mediated by the activation of ERK, indicating that the activation of this enzyme played a role in the proliferation of SFs. Lin et al (18) reported that atropine inhibited the activation of ERK and its downstream signaling molecule c-FOS in human retinal pigment epithelium (RPE) cells in vitro. Moreover, atropineinhibited myopia progression by downregulating the expression levels of retinal c-FOS in form-deprivation myopia (FDM). These results indicate that ERK signaling pathways in RPE cells and retinas play an important role in myopia progression. However, whether the expression of ERK $1 / 2$ in sclera is essential for the development of refraction remains unclear. Its underlying mechanisms also require further investigation. A large number of studies indicate that the inhibition of ERK1/2 downregulates the expression of MMP-2, which inhibits the invasion and migration of cancer cells (19-21). Given that the ERK1/2-MMP-2 pathway mediates the regulation of ECM in various tissues $(22,23)$ and that ocular elongation in myopia largely results from the remodeling of the scleral ECM, the current study examined whether the ERK1/2-MMP-2 pathway may affect the formation of FLM by regulating the remodeling of PS.

In the present study, guinea pigs were used to establish the FLM model. This species was selected since it is characterized by large pupils and reasonably large eyes (24). In addition, unlike chicken sclera, which has a cartilaginous layer and a fibrous layer, guinea pig sclera has only the fibrous layer, which is similar to human eyes (25). PD98059 is an organic compound inhibitor also known as 2-(2-amino-3-methoxyphenyl)-4H-1-benzopyran-4-one. It has been used to block the activity of ERK1/2 protein by inhibiting the activity of MEK both in vivo (26) and in vitro (27). In FDM rats, PD98059 abated the protective effect of levodopa on visual cortical neurons by inhibiting the protein levels of phosphorylated (p)-ERK1/2 (28).

The present study aimed to analyze the expression of MMP-2 and ERK1/2 in the PS of guinea pig models of FLM and to determine whether the ERK1/2-MMP-2 pathway was involved in the development of FLM by regulating the remodeling of PS.

\section{Materials and methods}

Animals. Guinea pigs (Cavia porcellus, English short-hair stock, tricolor strain; obtained from Jiagan Biological Co., Ltd.; n=40; age, 2 weeks; sex, male) were raised at a temperature of $25-27^{\circ} \mathrm{C}$ and a relative humidity of $55-65 \%$, with free access to food and water and to fresh vegetables supplied daily. All animals were maintained on a 12:12 h light-dark cycle prior to modeling. All animal experiments were in accordance with the ARVO Statement for the Use of Animals in Ophthalmic and Vision Research and complied with the ARRIVE guideline $(29,30)$.

Experimental design. A total of 40 guinea pigs were randomly divided into four groups ( $\mathrm{n}=10$ in each group). In the control group, animals were maintained on a 12-h light-dark cycle $(600$ lux; lights on 6:00 a.m.-6 p.m.) and both eyes of the guinea pigs received no treatment. In the FLM group, the guinea pigs were raised in specifically designed cages, as described in Luo et al (5), and were exposed to 600 lux illumination with a duty cycle of $50 \%$ at a flash rate of $0.5 \mathrm{~Hz}$ from 6:00 a.m. to 6 p.m. for 6 weeks. These conditions were used to induce FLM, as previously described by Luo et al (5). During housing, animals were monitored twice daily for health status, including food and water supply, general physical conditions, animal activities and eye conditions. No adverse events were observed. In the vehicle control group (FLM + DMSO), guinea pigs were administered a peribulbar injection of $100 \mu 10.1 \%$ dimethylsulfoxide (DMSO). In the FLM + PD98059 group, a peribulbar injection of $100 \mu$ l PD98059 [cat. no. HY-12028; MedChemExpress; dissolved in DMSO buffer at a concentration of $50 \mu \mathrm{M}$, as previously described (31)] was performed. Guinea pigs were anesthetized with isoflurane (RWD Life Science Inc.; cat. no. R510-22) prior to these injections. Anesthesia was induced with $4 \%$ isoflurane in oxygen for 2 min and then maintained with $2.5 \%$ isoflurane in oxygen for a further $2 \mathrm{~min}$. All peribulbar injections were administered to the right eyes every other day for 3 weeks, starting from the third week of flickering light exposure (10 times in total) (32). The experimenters were blinded to the pharmacological treatment while processing data and measuring refraction.

Measurements of refraction. Refraction was measured with streak retinoscopy (cat. no. KJ6B; Suzhou Kangjie Medical Co., Ltd.). Prior to the measurements of refraction, one drop of tropicamide ophthalmic solution (Santen Pharmaceutical Co., Ltd.) was administered four times every $5 \mathrm{~min}$ to achieve cycloplegia. The mean value of 3 readings was considered the final spherical equivalent (SE). All measurements were performed monocularly in the right eyes prior to and following 6 weeks of treatment with the assistance of an optometrist. The changes in refraction are presented as the difference in measurements between the 6th week and the baseline (week 0 ).

Tissue preparation. All guinea pigs were sacrificed with intraperitoneal injections with an overdose of pentobarbital sodium ( $200 \mathrm{mg} / \mathrm{kg}$ ) following the completion of the final refractive and axial measurements. After euthanasia and prior to the disposal of the animal carcasses, death was verified with a combination of criteria, including lack of pulse, breathing, corneal reflex and response to firm toe pinch, inability to detect respiratory sounds and heartbeat by use of a stethoscope, graying of the mucous membranes, and rigor mortis. The nasal and temporal limbi were marked and subsequently the anterior segments and the extraocular muscles were removed. The remaining tissues were cut into two parts from the markings on the frontal axis and fixed for $24 \mathrm{~h}$ at room temperature in specialized fixation solutions for eyeballs provided by the Eye and ENT Hospital of Fudan University (Shanghai, China). Every $100 \mathrm{ml}$ 
A

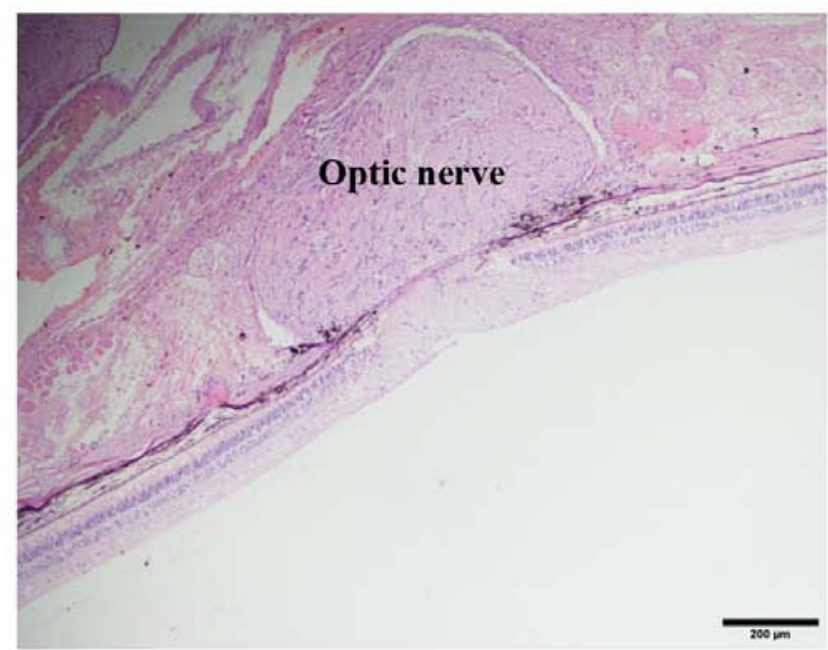

B

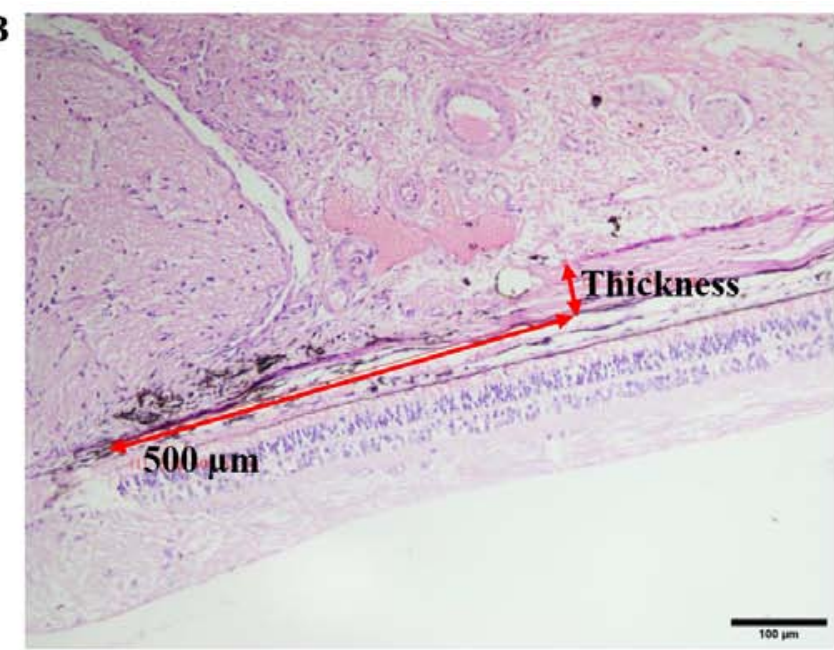

Figure 1. H\&E staining of PS. (A) A representative image of the optic nerve using the light microscope. (Scale bar: $200 \mu \mathrm{m}$. Magnification: 10x10) (B) Using the measuring tools on the microscope, a $500 \mu \mathrm{m}$ line segment was drawn starting at the centre of the optic nerve. Perpendicular to the segment, another line segment was drawn to measure the thickness of the PS. (Scale bar: $100 \mu \mathrm{m}$, magnification: x200). PS, posterior sclera.

fixation solutions, composed of $10 \mathrm{ml} 10 \%$ methanal, $85 \mathrm{ml}$ 95\% ethanol and $5 \mathrm{ml} 99 \%$ glacial acetic acid, is suitable for the fixation and preservation of the morphological structure of the eyeballs. Subsequently, the tissues were embedded in paraffin. The tissues of PS was collected from the back of the eye for western blot analysis and RT-qPCR assays (32). The internal part and the surface of the sclera were scraped to remove the vitreous body, choroid, retina and related extraocular tissues. All specimens were immediately frozen in liquid nitrogen to a temperature of $-198^{\circ} \mathrm{C}$ and subsequently stored at $-80^{\circ} \mathrm{C}$ until further analysis.

Thickness of PS. Paraffin sections (4- $\mu \mathrm{m}$ thick) were stained with $\mathrm{H} \& \mathrm{E}$ at room temperature (hematoxylin staining for $5 \mathrm{~min}$ and eosin staining for $1 \mathrm{~min}$ ). The sections were observed and images were captured under a light microscope (BH-2; Olympus Corporation). Scleral thickness was measured in the areas $0.5 \mathrm{~mm}$ from the optic nerve in the nasal and temporal planes of each sample, using the Cellsens Standard software (Version 1.12, Olympus Corporation) (33). The final thickness of the PS was expressed as the average of the nasal and temporal values (Fig. 1).

Immunohistochemical analysis. The paraffin slides from the aforementioned paraffin-embedded samples were dewaxed at $60^{\circ} \mathrm{C}$ for $30 \mathrm{~min}$ and rehydrated with xylene and graded alcohol solutions. Initially, the antigen of the tissues was retrieved in a microwave oven at a temperature of $95^{\circ} \mathrm{C}$ and subsequently the tissue sections were incubated in a $3 \% \mathrm{H}_{2} \mathrm{O}_{2}$ solution for $10 \mathrm{~min}$ at room temperature to inactivate endogenous peroxidase. Goat serum was used to block nonspecific binding for $15 \mathrm{~min}$ at room temperature. The slides were incubated with primary antibodies diluted to 1:200, including rabbit anti-MMP-2 polyclonal antibody (cat. no. NB200-193; Novus Biologicals, LLC) and rabbit anti-ERK1/2 monoclonal antibody (cat. no. 4695; Cell Signaling Technology, Inc.) at $4{ }^{\circ} \mathrm{C}$ overnight. A haopoly-HRP rat/rabbit secondary antibody (cat. no. JHB0030; Jie Hao Biotechnology Co., Ltd.) was added to the tissue sections the following morning for $1 \mathrm{~h}$ at room temperature. Subsequently, a 3,3'-diaminobenzidine chromogen kit was applied and the time was controlled under observation using a light microscope for $1.5 \mathrm{~min}$. Haematoxylin was used to stain the nucleus for $1 \mathrm{~min}$ at room temperature and the sample was washed with running water. Finally, following drying of the slides, they were sealed with resin and covered. PBS (0.01 M; pH 7.4) was applied instead of the primary antibody as a negative control.

Western blot analysis. Total scleral protein of the aforementioned fresh frozen samples was extracted using a tissue protein extraction kit (cat. no. CW0891M; CoWin Biosciences), containing phosphatase inhibitors (cat. no. BSP002-6; Sangon Biotech Co., Ltd.). Subsequently, the protein concentration was quantified by the Bradford method and the sample was boiled at $100^{\circ} \mathrm{C}$ for $10 \mathrm{~min}$ with a loading buffer (cat. no. 9173; Takara Bio, Inc.). Equal amounts of protein of $15 \mu \mathrm{g}$ per lane were separated using SDS-PAGE (10\% gel) and transferred onto a nitrocellulose membrane. Following blocking with $5 \%$ nonfat diary milk in Tris-buffered saline containing $0.05 \%$ Tween-20 (TBST) for $1 \mathrm{~h}$ at room temperature, the membrane was incubated with the following primary antibodies: Rabbit anti-MMP-2 (polyclonal antibody; 1:2,000; cat. no. NB200-193; Novus Biologicals, Ltd.), rabbit anti-ERK1/2 (monoclonal antibody; 1:1,000; cat. no. 4695; Cell Signaling Technology, Inc.) and rabbit anti-p-ERK1/2 (monoclonal antibody; 1:1,000; cat. no. 4370; Cell Signaling Technology, Inc.) at $4^{\circ} \mathrm{C}$ overnight. The same blots were stripped and reanalyzed using rabbit anti- $\beta$-tubulin antibody (polyclonal; 1:1,000; cat. no. 2146; Cell Signaling Technology, Inc.) as the internal control. The following morning, the membranes were washed four times with TBST for $10 \mathrm{~min}$ and incubated with a HRP-conjugated goat anti-rabbit IgG secondary antibody (1:5,000; cat. no. 01334/10146; CoWin Biosciences) for $1 \mathrm{~h}$. The signal was detected with a chemiluminescence kit (WBKLS0100; EMD Millipore) and an ECL detection system (ECL-Plus; Merck KGAa). Subsequently, the 
Table I. Values of the measurements of refraction.

\begin{tabular}{llll}
\hline Group & Week 0 (D) & Week 6 (D) & Week 6-week 0 (D) \\
\hline Control & $1.76 \pm 0.39$ & $-0.44 \pm 0.43$ & $-2.21 \pm 0.34$ \\
FLM & $1.72 \pm 0.62$ & $-6.03 \pm 0.76^{\mathrm{a}}$ & $-7.75 \pm 0.48^{\mathrm{a}}$ \\
FLM + DMSO & $1.79 \pm 0.28$ & $-5.89 \pm 0.53$ & $-7.68 \pm 0.46$ \\
FLM + PD98059 & $1.76 \pm 0.71$ & $-3.92 \pm 0.70^{\mathrm{b}}$ & $-5.68 \pm 0.29^{\mathrm{c}}$
\end{tabular}

All data are presented as the mean \pm SEM. ${ }^{a} \mathrm{P}<0.001$ compared with the control group. ${ }^{b} \mathrm{P}<0.05$ compared with the FLM + DMSO group. ${ }^{\mathrm{c}} \mathrm{P}<0.01$ compared with the FLM + DMSO group. FLM, flickering light-induced myopia; DMSO, dimethylsulfoxide; D, diopter.

bands were semi-quantified using a Bio-Rad 2000 gel imaging system with Quantity One software (version 4.6.6; Bio-Rad Laboratories, Inc.).

RNA extraction and reverse transcription quantitative $P C R(R T-q P C R)$. Total RNA was extracted from the aforementioned fresh frozen tissues using the TRIzol reagent (cat. no. 15596-026; Thermo Fisher Scientific Inc.) and a tissue RNA purification kit (ES-RN002plus; Shanghai Yishan Biotechnology Co., Ltd.). Subsequently, the RNA was reverse transcribed to cDNA using the PrimeScript ${ }^{\mathrm{TM}}$ RT Master Mix kit (Takara Bio, Inc.) according to the manufacturer's instructions. RT-qPCR analysis was performed on SDS 7300 instrument (Applied Biosystems; Thermo Fisher Scientific, Inc.) with $\mathrm{TB}$ Green Premix Ex $\mathrm{Taq}^{\mathrm{TM}}$ (cat. no. RR420A; Takara Bio, Inc.). The primer sequences used for the PCR were as follows: MMP-2 forward, 5'-AATGGCCGCGAA TACACCAG-3' and reverse, 5'-AATGGCCGCGAATACACC AG-3'; $\beta$-tubulin forward, 5'-GTTCGATGCCCGCAATAC CA-3' and reverse, 5'-CATCTTGCCCCGGTAGATGC-3'. The qPCR conditions were as follows: $95^{\circ} \mathrm{C}$ for $30 \mathrm{sec}$; 40 cycles of $95^{\circ} \mathrm{C}$ for $5 \mathrm{sec}$ and $60^{\circ} \mathrm{C}$ for $31 \mathrm{sec}$. Melting curves were used to assess and confirm the specificity of the products generated for each set of primers. Following amplification, the $2^{-\Delta \Delta \mathrm{Cq}}$ comparative method was used to analyze gene expression levels (34). The experiments were performed in triplicate.

Statistical analysis. All data were analyzed with the SPSS 22.0 software (IBM Corp.) and presented as the mean \pm SEM. All the experiments were repeated three times. The differences among groups were assessed by one-way ANOVA followed by Bonferroni's multiple comparisons test. $\mathrm{P}<0.05$ was considered to indicate a statistically significant difference.

\section{Results}

Measurement of refraction in the right eyes prior to and following modeling. The values of refraction in the right eyes in all groups prior to and following modeling are shown in Table I. Prior to the treatments, no significant differences in refraction of the right eyes were observed among the groups ( $n=10 /$ group; all $\mathrm{P}>0.05$ ). Following 6 weeks of treatment, refraction in the FLM group was significantly decreased compared with that of the control group $(\mathrm{P}<0.001)$. Refraction of the right eyes at week 6 in the FLM + PD98059 group was higher than that noted in the FLM + DMSO group $(\mathrm{P}=0.031)$. Moreover, the changes of refraction between weeks 0 and 6 indicated that the FLM group developed significant myopia compared with the control group $(\mathrm{P}<0.001)$. Administration of PD98059 inhibited the changes in refraction compared with those of the FLM + DMSO group $(\mathrm{P}=0.001)$.

Morphological changes and thickness measurement of the PS. The PS was stained with H\&E and the images were obtained using light microscopy. The PS tissues consisted of a large number of SFs and fiber bundles. The PS of the FLM group exhibited a higher number of loose and disordered collagen fibers than that of the control group. The collagenous fiber bundles of the PS were thinner in the FLM group than those in the control group. However, the FLM + PD98059 group inhibited the changes in PS remodeling compared with those of the FLM + DMSO group. Moreover, the PS thickness was measured using the measuring tools on the microscope. Firstly, a $500 \mu \mathrm{m}$ line segment was drawn starting at the center of the optic nerve. Then, perpendicular to the segment, another line segment was drawn to measure the thickness of the PS (Fig. 1). The PS thickness was decreased in the FLM group compared with that of the control group $(64.76 \pm 2.41 \mathrm{vs}$. $132.78 \pm 8.52 \mu \mathrm{m} ; \mathrm{P}<0.001$; both $\mathrm{n}=4$ ), while $\mathrm{PD} 98059$ partially inhibited the decreasing trend in the FLM + PD98059 group $(n=3)$ compared with that of the FLM + DMSO group $(n=4)$ (105.79 \pm 1.77 vs. $66.05 \pm 2.44 \mu \mathrm{m} ; \mathrm{P}=0.001)$ (Fig. 2).

Immunohistochemical staining of MMP-2 and ERK1/2. As shown in Fig. 3, MMP-2 was mainly localized in the cytoplasm of SFs, scleral extracellular matrix, outer plexiform layer, inner nuclear layer (INL) and retinal ganglion cells (RGCs). MMP-2 was colocalized with ERK1/2, which was also mainly found in the cytoplasm of SFs, INL and RGCs (Fig. 3).

Determination of MMP-2 and ERK1/2 expression levels in the sclera. The results of the RT-qPCR analysis are shown in Fig. 4. The mRNA expression levels of MMP-2 in the FLM group were significantly higher than those of the control group $(\mathrm{P}=0.043 ; \mathrm{n}=3)$. Moreover, MMP-2 mRNA levels were significantly downregulated in the FLM + PD98059 group compared with those of the FLM+DMSO group $(\mathrm{P}=0.011 ; \mathrm{n}=3)$. The results of the western blot analysis are shown in Fig. 5. The protein levels of MMP-2 and the activation levels of ERK1/2 (p-ERK/ERK) in the FLM group were significantly higher than those of the control group $(\mathrm{P}=0.011$ and $\mathrm{P}<0.001$; both $\mathrm{n}=5)$. In addition, the $\mathrm{p}-\mathrm{ERK} / \mathrm{ERK}$ ratio of the FLM + PD98059 group 
A
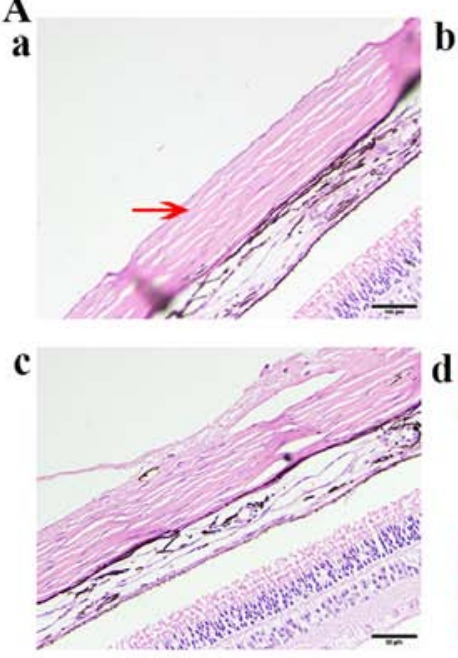

b

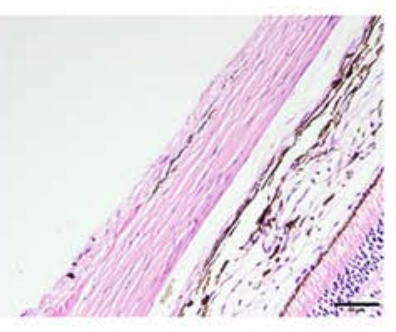

d

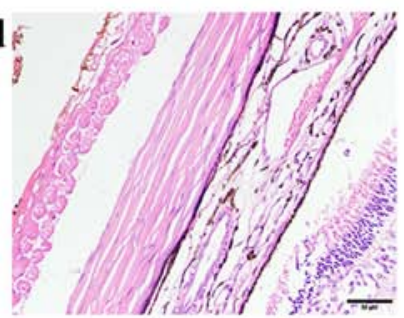

B

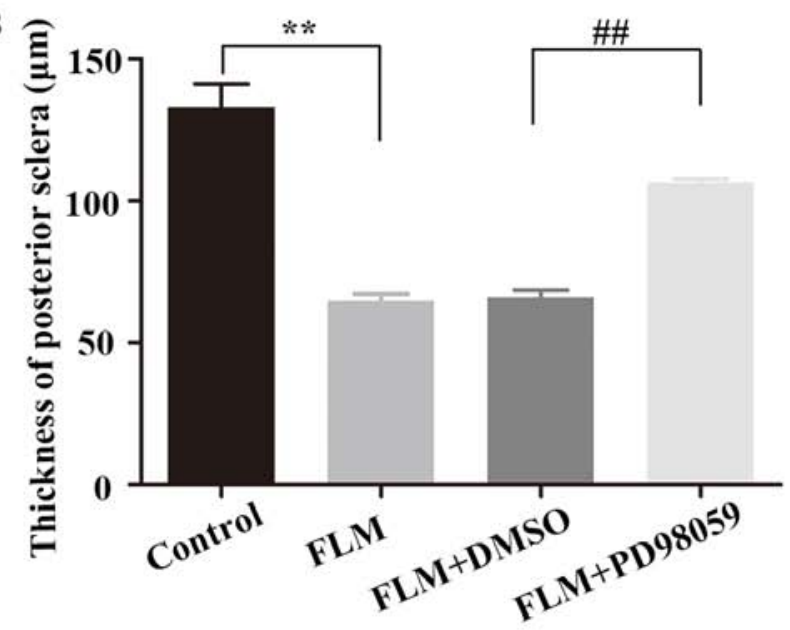

Figure 2. Morphological changes in PS. (A) H\&E staining of the structure of the eyeballs in the four groups, including (A-a) Control, (A-b) FLM, (A-c) FLM + DMSO and (A-d) FLM + PD98059. The red arrow indicates the PS. Scale bar, $50 \mu \mathrm{m}$; Magnification, $\mathrm{x} 400$ ). (B) Thickness of the PS. ${ }^{* *} \mathrm{P}<0.01$ compared with the control group. ${ }^{\# \#} \mathrm{P}<0.01$ compared with the FLM + DMSO group. PS, posterior sclera; FLM, flickering light-induced myopia; DMSO, dimethylsulfoxide.

A

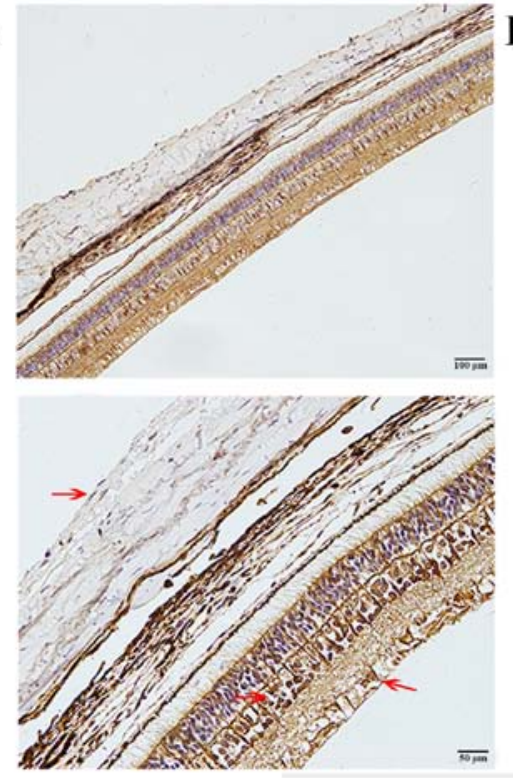

B

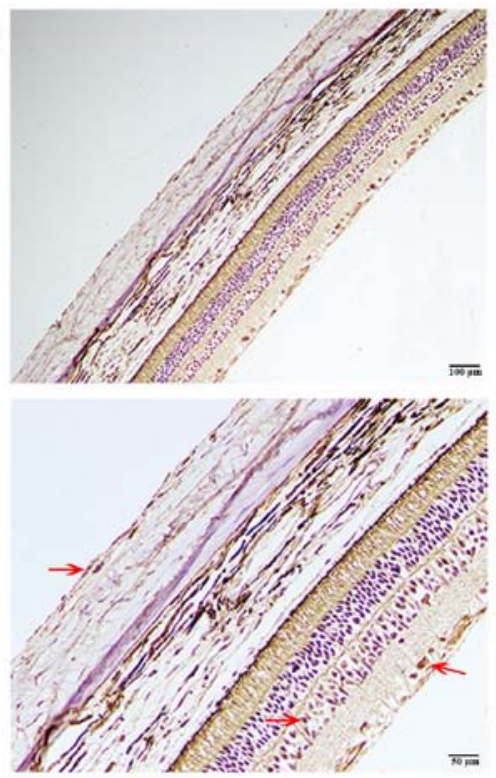

Figure 3. Immunohistochemical staining of MMP-2 and ERK1/2. (A) Immunohistochemical staining of ERK1/2. (B) Immunohistochemical staining of MMP2. Brown staining (red arrows) represents the location of MMP-2 or ERK1/2 and blue staining represents the cell nucleus (scale bar, $100 \mu \mathrm{m}$ for the top row and $50 \mu \mathrm{m}$ for the bottom row; magnification, $\mathrm{x} 200$ for the top row and $\mathrm{x} 400$ for the bottom row). ERK1/2 was mainly found in the cytoplasm of SFs, INL and RGCs. MMP-2 was mainly localized in the cytoplasm and the extracellular matrix of SFs, INL and RGCs. SFs, scleral fibroblasts; INL, inner nuclear layer; RGCs, retinal ganglion cells.

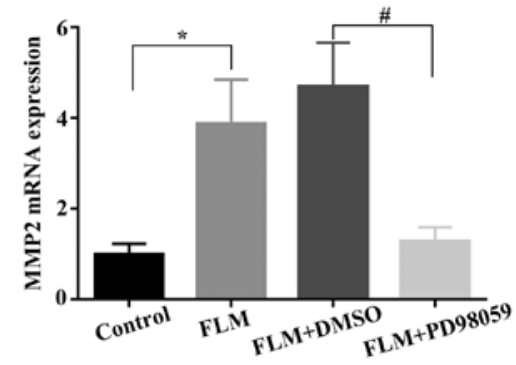

Figure 4. mRNA expression levels of MMP-2. "P<0.05 compared with the control group. ${ }^{\#} \mathrm{P}<0.05$ compared with the FLM + DMSO group. FLM, flickering light-induced myopia; DMSO, dimethylsulfoxide. was decreased compared with that of the FLM + DMSO group $(\mathrm{P}=0.028$; both $\mathrm{n}=5)$. Furthermore, decreased MMP-2 expression was observed in the FLM + PD98059 group compared with that of the FLM + DMSO group $(\mathrm{P}=0.001$; both $\mathrm{n}=5)$.

\section{Discussion}

In the present study, myopia was induced in 2-week-old guinea pigs by exposure to flickering light $(0.5 \mathrm{~Hz})$. The results indicated that flickering light could induce a myopic shift in refraction. The changes in refraction over time were 

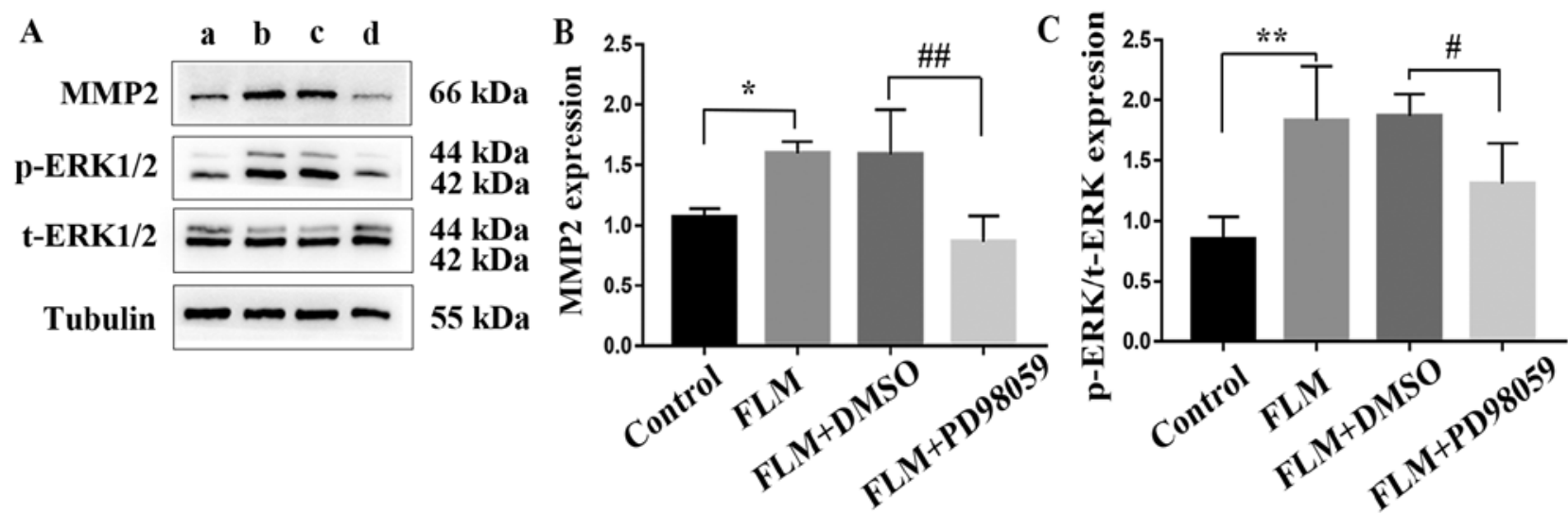

Figure 5. Western blot analysis of MMP-2, p-ERK1/2 and ERK1/2 expression in each group. (A) Representative western blot images. (a) lane: Control group; (b) lane: FLM group; (c) lane: FLM+DMSO group; (d) lane: FLM+PD98059 group. (B) MMP-2 and (C) p-ERK/ERK protein levels. "P<0.05 and ${ }^{* * *} \mathrm{P}<0.01$ compared with the control group. ${ }^{\#} \mathrm{P}<0.05$ and ${ }^{\# \#} \mathrm{P}<0.01$ compared with the FLM + DMSO group. FLM, flickering light-induced myopia; DMSO, dimethylsulfoxide; $p$, phosphorylated; $\mathrm{t}$, total.

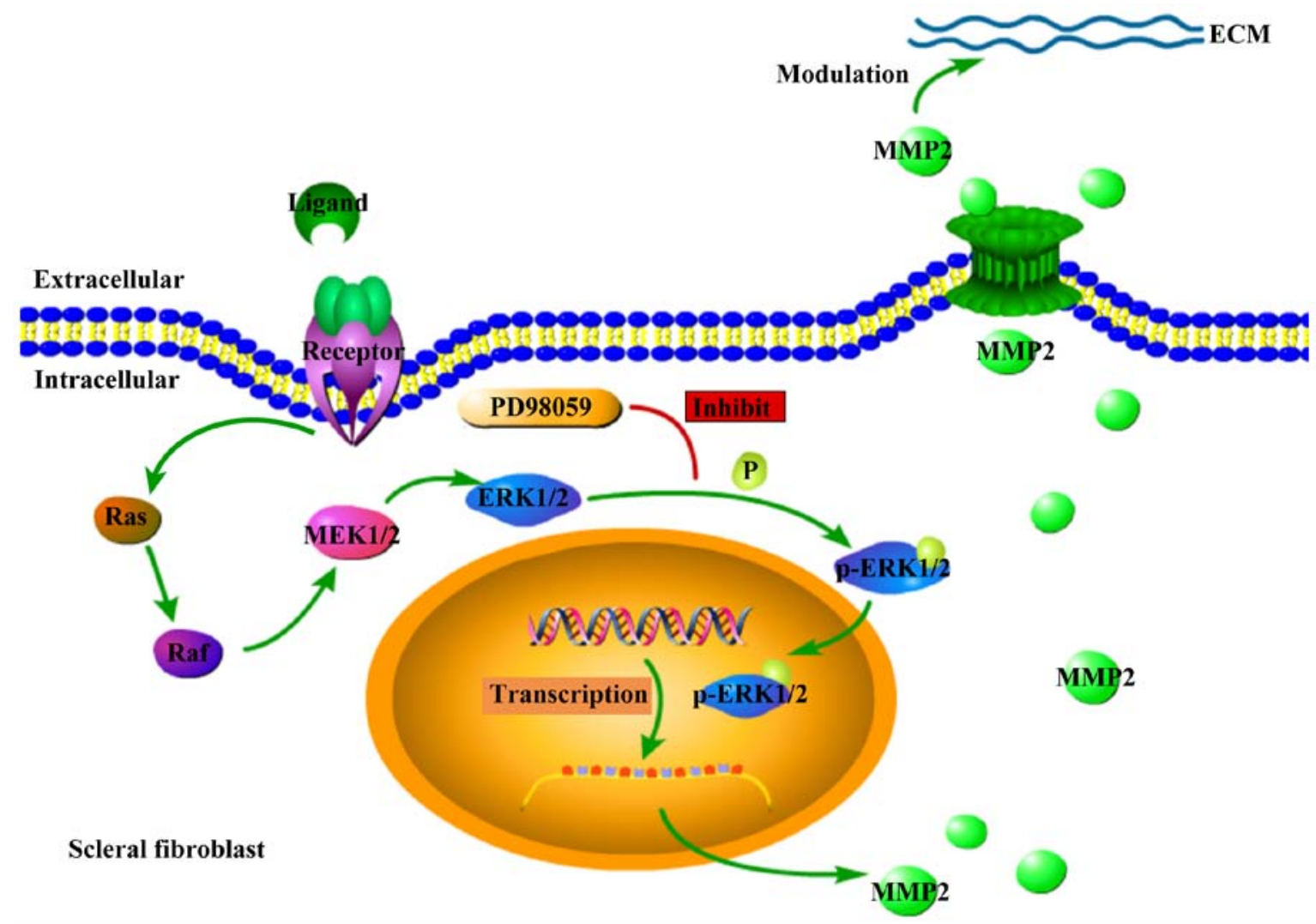

Figure 6. Diagram of ERK1/2-MMP2 pathway involved in scleral fibroblast in FLM. Following extracellular ligand binding to the corresponding receptors, the intracellular RAS-RAF-MEK1/2-ERK1/2 pathway was activated, leading to phosphorylation of ERK1/2 and subsequent upregulation of transcription and translation of MMP-2. Then the upregulated expression of MMP-2 caused excessive degradation in modulating the ECM. Following the PD98059 peribulbar injection to the animals, the phosphorylation of ERK1/2 was inhibited and therefore the expression of MMP-2 was downregulated. This resulted in the inhibition of the development of FLM. ECM, extracellular matrix; FLM, flickering light-induced myopia.

markedly increased in the FLM group compared with those noted in the control group. However, the FLM + PD98059 group exhibited significantly decreased changes in refraction in the right eyes compared with those of the FLM + DMSO group, which indicated that PD98059 could partly inhibit FLM. The present study was in accordance with a previous study that reported the induction of myopia by flickering light (0.5 Hz) (4-6).
In addition, the right eyes of guinea pigs were used to investigate the effects of flickering light on the induction of myopia for two main reasons. Firstly, both eyes of the same animal in the FLM group were exposed to flickering light and they were susceptible to the same environmental light disturbance. Consequently, both eyes exhibited the same tendency to develop nearsightedness. In addition, anisometropia may induce an interference effect between the experimental eyes 
and the fellow eyes (35). Moreover, the interruption of binocular fusion and the normal binocular fixation pattern induces anisometropia, which is associated with both amblyopia and strabismus. Various studies have focused on refractive development but rarely provided data on sensory or oculomotor development (36). Therefore, to minimize autointerference, a comparison was performed between pre- and post-treatment values.

In the present study, the PS structures in the FLM group were morphologically altered in guinea pigs. The FLM group exhibited a looser arrangement of collagen fibers and a thinner tissue structure than the control group, which was consistent with previous findings in humans and other experimental myopia models. During myopia development, scleral remodeling, including biomechanical changes, contributes to axial elongation (37). Patients with high myopia exhibit thinner scleral collagen fibrils compared with the eyes of healthy subjects $(38,39)$. In an FDM model, scleral tissue loss and scleral thinning were observed in tree shrews during modeling (40). In the lens-induced myopia model, the elastic modulus and crimp angle of collagen fibrils were associated with the axial elongation rate during monocular minus lens wearing and recovery (41). MMP-2 may play a role in the process of PS remodeling in myopia. MMP-2 is a member of the MMP family. These proteins are produced by SFs, secreted into the ECM of sclera and are capable of degrading and altering the scleral ECM, including proteoglycans and collagens (42). A large number of studies have reported that upregulated MMP-2 expression is involved in the formation and development of myopia, both in human eyes $(13,14,43)$ and in animal models $(8,12,44,45)$. Li et al (46) indicated that retinal MMP-2 levels were upregulated, while TIMP2 levels were downregulated in FLM mice at 1 or 2 weeks of treatment. Di (47) observed an increasing tendency in scleral MMP-2 expression of guinea pigs following 3 weeks of FLM modeling. These results indicate that MMP-2 expression may be important in the formation of FLM. In accordance with the former findings, the present study demonstrated that MMP-2 was localized in the cytoplasm of fibroblasts and in the extracellular matrix of sclera, while its expression was upregulated following 6 weeks of FLM modeling. This evidence supports the hypothesis that MMP-2 may participate in the PS remodeling during the development of FLM.

Notably, in the present study, the results indicated that ERK1/2 was colocalized with MMP-2 in guinea pig eyes. In addition, the protein levels of p-ERK1/2 and MMP-2 were increased in the FLM group compared with those of the control group. Following peribulbar injections of PD98059, a specific inhibitor of the ERK1/2 protein kinase, to the guinea pigs, the increased MMP-2 expression was attenuated at both the mRNA and protein levels. Moreover, the remodeling of PS was partly reversed and the changes in refraction were inhibited, indicating that MMP-2 may be a downstream target of ERK1/2. In human SFs, ERK1/2 is expressed in the cytoplasm and its activation is associated with proliferation (48). In line with this evidence, the present study demonstrated that ERK1/2 was expressed in the cytoplasm of SFs. ERK1/2 is one of the members of the MAPK signaling pathway, which also includes the p38 and c-JNK pathways. Wang et al (49) demonstrated that the expression levels of p38 MAPK were increased in scleral tissues in red flickering light-induced myopia compared with the natural light-exposed eyes, suggesting that MAPK may play an important role in myopia development. In addition, the ERK signaling pathway was activated and was involved in the emmetropization process in chicks (50). Moreover, it has been shown that cyclic adenosine monophosphate (cAMP) regulates collagen synthesis in human SFs via ERK1/2 phosphorylation and that CAMP agonists can reduce collagen synthesis and induce myopia (51). Therefore, it was concluded that the activation of p-ERK1/2 was involved in collagen synthesis and myopia development (51).

The ERK1/2-MMP-2 pathway is involved in the proliferation and migration of cancer cells (52-54). However, less is known regarding its function in the modulation of sclera. It has been shown that the binding of stimuli, such as growth factors or cytokines to their corresponding ligands activates the phosphorylation of ERK1/2 and, subsequently, p-ERK1/2 is transported into the nucleus, which mediates the transcription of the downstream genes (15). Therefore, it was hypothesized that guinea pigs raised under flickering light would undergo a series of signaling events that would stimulate the activation of the ERK1/2 pathway in SFs. Following these events, p-ERK1/2 would be transported into the nucleus, regulating the expression of MMP-2 and finally remodeling the PS leading to FLM development. Following treatment with PD98059, the activation of the ERK1/2-MMP-2 pathway was inhibited. Therefore, the development of FLM was suppressed (Fig. 6). In addition, in a previous study, the application of dopamine (DA) or quinpirole transiently activated ERK during the day, whereas it caused sustained inhibition of ERK during the night (55), indicating that the DA-ERK pathway was associated with light conditions and circadian rhythms. Moreover, our previous study indicated that the retinal and choroidal DA levels in guinea pigs were upregulated and associated with FLM (5). Therefore, it may be hypothesized that the equilibrium state of photoreceptors in retina is disturbed by flickering light, so that DA levels are upregulated. This in turn may affect the scleral ERK1/2-MMP-2 pathway. This hypothesis will be further studied in the future by our research group.

In conclusion, the data indicated that the peribulbar delivery of PD98059 inhibited the changes in refraction of guinea pigs with FLM via the ERK1/2-MMP-2 pathway by abating the remodeling of PS. Therefore, these results revealed a signaling mechanism involved in FLM development and a potential molecular target for preventing or treating myopia. However, the mechanism by which PD98059 prevents myopia requires further studies in other experimental models. Additional work is also needed to determine the upstream and downstream molecules of this pathway.

\section{Acknowledgements}

Not applicable.

\section{Funding}

This work was supported by the Natural Science Foundation of Shanghai (grant no. 17ZR1404200), Shanghai Municipal Commission of Health and Family Planning 
(grant no. 201640046); and the Shanghai Municipal Science and Technology Commission (grant no. 17411950203).

\section{Availability of data and materials}

The datasets used and/or analyzed during the current study are available from the corresponding author on reasonable request.

\section{Authors' contributions}

MS wrote the manuscript and performed the experiments. BL designed the study and critically revised the manuscript. MS and BL confirm the authenticity of all the raw data. QH and TL analyzed and interpreted the results. XZ designed the experiments and approved the final version of the manuscript. All authors read and approved the final manuscript.

\section{Ethics approval and consent to participate}

The experimental procedure and protocols were approved by the Ethics Approval Committee of the Jinshan Hospital of Fudan University (Shanghai, China).

\section{Patient consent for publication}

Not applicable.

\section{Competing interests}

The authors declare that they have no competing interests.

\section{References}

1. Smolensky MH, Sackett-Lundeen LL and Portaluppi F: Nocturnal light pollution and underexposure to daytime sunlight: Complementary mechanisms of circadian disruption and related diseases. Chronobiol Int 32: 1029-1048, 2015.

2. Cremieux J, Orban GA, Duysens J, Amblard B and Kennedy H: Experimental myopia in cats reared in stroboscopic illumination. Vision Res 29: 1033-1036, 1989.

3. Yu Y, Chen H, Tuo J and Zhu Y: Effects of flickering light on refraction and changes in eye axial length of C57BL/6 mice. Ophthalmic Res 46: 80-87, 2011.

4. Li B, Luo X, Li T, Zheng C, Ji S, Ma Y, Zhang S and Zhou X: Effects of constant flickering light on refractive status, 5-HT and 5-HT2A receptor in guinea pigs. PLoS One 11: e167902, 2016.

5. Luo X, Li B, Li T, Di Y, Zheng C, Ji S, Ma Y, Zhu J, Chen X and Zhou X: Myopia induced by flickering light in guinea pig eyes is associated with increased rather than decreased dopamine release. Mol Vis 23: 666-679, 2017.

6. Di Y, Liu R, Chu R, Zhou X and Zhou X: Establishment of a guinea pig model of light perception myopia induced by flickering light. Acta Lab Anim Sci Sin 20: 48-52, 2012.

7. Harper AR and Summers JA: The dynamic sclera: Extracellular matrix remodeling in normal ocular growth and myopia development. Exp Eye Res 133: 100-111, 2015.

8. Zhao F, Zhou Q, Reinach PS, Yang J, Ma L, Wang X, Wen Y, Srinivasalu N, Qu J and Zhou X: Cause and effect relationship between changes in scleral matrix metallopeptidase-2 expression and myopia development in mice. Am J Pathol 188: 1754-1767, 2018.

9. Yang SR, Ye JJ and Long Q: Expressions of collagen, matrix metalloproteases-2, and tissue inhibitor of matrix metalloproteinase-2 in the posterior sclera of newborn guinea pigs with negative lens-defocused myopia. Zhongguo Yi Xue Ke Xue Yuan Xue Bao 32: 55-59, 2010 (In Chinese).
10. Liu HH, Kenning MS, Jobling AI, McBrien NA and Gentle A: Reduced scleral TIMP-2 expression is associated with myopia development: TIMP-2 supplementation stabilizes scleral biomarkers of myopia and limits myopia development. Invest Ophthalmol Vis Sci 58: 1971-1981, 2017.

11. Deng H, Tian Y, Zhou X, Zhang X and Meng J: Effect of bilberry extract on development of form-deprivation myopia in the guinea Pig. J Ocul Pharmacol Ther 32: 196-202, 2016.

12. Schippert R, Brand C, Schaeffel F and Feldkaemper MP: Changes in scleral MMP-2, TIMP-2 and TGFbeta-2 mRNA expression after imposed myopic and hyperopic defocus in chickens. Exp Eye Res 82: 710-719, 2006.

13. Jia Y, Hu DN, Sun J and Zhou J: Correlations between MMPs and TIMPs levels in aqueous humor from high myopia and cataract patients. Curr Eye Res 42: 600-603, 2017.

14. Zhuang H, Zhang R, Shu Q, Jiang R, Chang Q, Huang X, Jiang C and Xu G: Changes of TGF- $\beta 2$, MMP-2, and TIMP-2 levels in the vitreous of patients with high myopia. Graef Arch Clin Exp 252: 1763-1767, 2014.

15. Yao B, Wang S, Xiao P, Wang Q and Zhang Y: MAPK signaling pathways in eye wounds: Multifunction and cooperation. Exp Cell Res 359: 10-16, 2017

16. Xie J, Zhang $\mathrm{Z}$ and Yin $\mathrm{H}$ : Investigation of transcripts of ERK mRNAs in developmental visual cortex of rats. Chin J Neuroanatomy 17: 309-314, 2001.

17. Barathi VA, Weon SR and Beuerman RW: Expression of muscarinic receptors in human and mouse sclera and their role in the regulation of scleral fibroblasts proliferation. Mol Vis 15: 1277-1293, 2009.

18. Lin H, Wei C, Chang C, Chen T, Hsu Y, Hsieh Y, Chen H and Wan L: Role of chronic inflammation in myopia progression: Clinical evidence and experimental validation. EBioMedicine 10: 269-281, 2016.

19. Gong J, Zhu S, Zhang Y and Wang J: Interplay of VEGFa and MMP2 regulates invasion of glioblastoma. Tumor Biol 35: 11879-11885, 2014

20. Yu S and Kim SJ: Salinomycin causes migration and invasion of human fibrosarcoma cells by inducing MMP-2 expression via PI3-kinase, ERK-1/2 and p38 kinase pathways. Int J Oncol 48: 2686-2692, 2016.

21. Chen F, Deng J, Liu X, Li W and Zheng J: HCRP-1 regulates cell migration and invasion via EGFR-ERK mediated up-regulation of MMP-2 with prognostic significance in human renal cell carcinoma. Sci Rep 5: 13470, 2015.

22. Elliot SJ, Catanuto P, Espinosa-Heidmann DG, Fernandez P, Hernandez E, Saloupis P, Korach K, Karl M and Cousins SW: Estrogen receptor $\beta$ protects against in vivo injury in RPE cells. Exp Eye Res 90: 10-16, 2010.

23. Song H, Cheng Y, Bi G, Zhu Y, Jun W, Ma W and Wu H: Release of matrix metalloproteinases- 2 and 9 by S-Nitrosylated Caveolin-1 contributes to degradation of extracellular matrix in tPA-Treated Hypoxic Endothelial Cells. PLoS One 11: e149269, 2016.

24. Howlett MH and McFadden SA: Spectacle lens compensation in the pigmented guinea pig. Vision Res 49: 219-227, 2009.

25. Marzani D and Wallman J: Growth of the two layers of the chick sclera is modulated reciprocally by visual conditions. Invest Ophthalmol Vis Sci 38: 1726-1739, 1997.

26. Huang P, Sun Y, Yang J, Chen S, Liu AD. Holmberg L, Huang X, Tang $\mathrm{C}$, Du J and Jin $\mathrm{H}$ : The ERK1/2 signaling pathway is involved in sulfur dioxide preconditioning-induced protection against cardiac dysfunction in isolated perfused rat heart subjected to myocardial ischemia/reperfusion. Int J Mol Sci 14: 22190-22201, 2013.

27. Tsang F, Koh AH, Ting WL, Wong PT and Wong WS: Effects of mitogen-activated protein kinase kinase inhibitor PD 098059 on antigen challenge of guinea-pig airways in vitro. $\mathrm{Br}$ J Pharmacol 125: 61-68, 1998.

28. Sun XN, Wang HL, Qiao G, Tao J, Liu C and Hao XH: The protective effect of the activation of NMDAR1/ERK1/2 signal pathway induced by levodopa on visual cortical neurons in monocular deprivation rats. Zhonghua Yan Ke Za Zhi 53: 931-940, 2017 (In Chinese).

29. National Research Council (US): Institute for Laboratory Animal Research. Guide for the Care and Use of Laboratory Animals. The National Academies Press (US), Washington, DC, 2011.

30. Percie du Sert N, Ahluwalia A, Alam S, Avey MT, Baker M, Browne WJ, Clark A, Cuthill IC, Dirnagl U, Emerson M, et al: Reporting animal research: Explanation and elaboration for the ARRIVE guidelines 2.0. PLoS Biol 18: e3000411, 2020. 
31. Alessi DR, Cuenda A, Cohen P, Dudley DT and Saltiel AR: PD 098059 is a specific inhibitor of the activation of Mitogen-activated protein kinase Kinase in vitro and in vivo. J Biol Chem 270: 27489-27494, 1995.

32. Tian XD, Cheng YX, Liu GB, Guo SF, Fan CL, Zhan LH and $\mathrm{Xu}$ YC: Expressions of type I collagen, $\alpha 2$ integrin and $\beta 1$ integrin in sclera of guinea pig with defocus myopia and inhibitory effects of bFGF on the formation of myopia. Int J Ophthalmol 6 : $54-58,2013$.

33. Zou L, Liu R, Zhang X, Chu R, Dai J, Zhou H and Liu H: Upregulation of regulator of G-protein signaling 2 in the sclera of a form deprivation myopic animal model. Mol Vis 20: 977-987, 2014.

34. Livak KJ and Schmittgen TD: Analysis of relative gene expression data using real-time quantitative PCR and the 2(-Delta Delta C(T)) method. Methods 25: 402-408, 2001.

35. Vincent SJ, Collins MJ, Read SA and Carney LG: Myopic anisometropia: Ocular characteristics and aetiological considerations. Clin Exp Optom 97: 291-307, 2014.

36. Smith ER, Hung LF, Arumugam B, Wensveen JM, Chino YM and Harwerth RS: Observations on the relationship between anisometropia, amblyopia and strabismus. Vision Res 134: 26-42, 2017.

37. Levy AM, Fazio MA and Grytz R: Experimental myopia increases and scleral crosslinking using genipin inhibits cyclic softening in the tree shrew sclera. Ophthal Physl Opt 38: 246-256, 2018.

38. Liu KR, Chen MS and Ko LS: Electron microscopic studies of the scleral collagen fiber in excessively high myopia. Taiwan Yi Xue Hui Za Zhi 85: 1032-1038, 1986.

39. Curtin BJ, Iwamoto T and Renaldo DP: Normal and staphylomatous sclera of high myopia. An electron microscopic study. Arch Ophthalmol 97: 912-915, 1979.

40. McBrien NA, Cornell LM and Gentle A: Structural and ultrastructural changes to the sclera in a mammalian model of high myopia. Invest Ophthalmol Vis Sci 42: 2179-2187, 2001.

41. Grytz R and Siegwart JJ: Changing material properties of the tree shrew sclera during minus lens compensation and recovery. Invest Ophthalmol Vis Sci 56: 2065-2078, 2015.

42. Sternlicht MD and Werb Z: How matrix metalloproteinases regulate cell behavior. Annu Rev Cell Dev Biol 17: 463-516, 2001

43. Jia Y, Hu DN, Zhu D, Zhang L, Gu P, Fan X and Zhou J: MMP-2, MMP-3, TIMP-1, TIMP-2, and TIMP-3 protein levels in human aqueous humor: Relationship with axial length. Invest Ophthalmol Vis Sci 55: 3922-3928, 2014.

44. Chen M, Qian Y, Dai J and Chu R: The sonic hedgehog signaling pathway induces myopic development by activating matrix metalloproteinase (MMP)-2 in Guinea pigs. PLoS One 9 e96952, 2014.
45. Xi LY, Yip SP, Shan SW, Summers-Rada J and Kee CS: Region-specific differential corneal and scleral mRNA expressions of MMP2, TIMP2, and TGFB2 in highly myopic-astigmatic chicks. Sci Rep 7: 11423, 2017.

46. Li M, Yu Y, Guan H and Chen H: The modulation of retinal Egr-1 and the extracellular matrix remodeling pathway in flickering light-induced myopioa in mice. Chin J Optom Ophthalmol 16: 328-334, 2014.

47. Di Y: Effects of stroboscopic illumination on emmetropization of guinea pig eye. Shanghai, Fudan University, 2013.

48. Huo L, Cui D, Yang X, Gao Z, Trier K and Zeng J: All-trans retinoic acid modulates mitogen-activated protein kinase pathway activation in human scleral fibroblasts through retinoic acid receptor beta. Mol Vis 19: 1795-1803, 2013.

49. Wang H, Zhuang K, Gao L, Zhang L and Yang H: Increased expression of CCN2 in the red flashing light-induced myopia in guinea pigs. Biomed Res Int 2013: 761823, 2013.

50. Penha AM, Burkhardt E, Schaeffel F and Feldkaemper MP: Effects of intravitreal insulin and insulin signaling cascade inhibitors on emmetropization in the chick. Mol Vis 18: 2608-2622, 2012.

51. Tao Y, Pan M, Liu S, Fang F, Lu R, Lu C, Zheng M, An J, Xu H, Zhao F, et al: cAMP level modulates scleral collagen remodeling, a critical step in the development of myopia. PLoS One 8: e71441, 2013.

52. Deng R, Mo F, Chang B, Zhang Q, Ran H, Yang S, Zhu Z, $\mathrm{Hu} \mathrm{L}$ and Su Q: Glucose-derived AGEs enhance human gastric cancer metastasis through RAGE/ERK/Sp1/MMP2 cascade. Oncotarget 8: 104216-104226, 2017.

53. Guan H, Guo Z, Liang W, Li H, Wei G, Xu L, Xiao H and Li Y. Trop2 enhances invasion of thyroid cancer by inducing MMP2 through ERK and JNK pathways. BMC Cancer 17: 486, 2017.

54. Han Y, Wu Z, Wu T, Huang Y, Cheng Z, Li X, Sun T, Xie X, Zhou Y and Du Z: Tumor-suppressive function of long noncoding RNA MALAT1 in glioma cells by downregulation of MMP2 and inactivation of ERK/MAPK signaling. Cell Death Dis 7: e2123, 2016.

55. Ko GY, Ko ML and Dryer SE: Circadian phase-dependent modulation of cGMP-gated channels of cone photoreceptors by dopamine and D2 agonist. J Neurosci 23: 3145-3153, 2003.

This work is licensed under a Creative Commons Attribution-NonCommercial-NoDerivatives 4.0 International (CC BY-NC-ND 4.0) License. 